\title{
Fuzzy logic controller design for PUMA 560 robot manipulator
}

\author{
Abdel-Azim S. Abdel-Salam, Ibrahim N. Jleta \\ Department of Electrical and Computer Engineering, The Libyan Academy, Libya
}

\begin{tabular}{l}
\hline Article Info \\
\hline Article history: \\
Received Oct 1, 2019 \\
Revised Jan 13, 2020 \\
Accepted Feb 11, 2020 \\
\hline Keywords: \\
Fuzzy logic controller (FLC) \\
PID-computed torque controller \\
(PID-CTC) \\
PUMA 560 robot manipulator
\end{tabular}

\begin{abstract}
The dynamic model of the robot manipulator contain from equations, these equations are nonlinear and contained from variations parameters due to variations in load, friction, and disturbance. The conventional computed torque (PD and PID) controllers are not highly suitable for nonlinear, complex, time-variant systems with delay. In this paper, the fuzzy logic controllers (FLC) has been used because it is efficient tools for control of nonlinear and uncertain parameters systems. This paper aims to design a fuzzy logic controller for position control of a PUMA 560 robot manipulator. Based on simulation results we conclude that the performance of the fuzzy logic controller in term of position tracking error in case of disturbance or load is better than the conventional computed torque (PD-CTC and PID-CTC) controllers.
\end{abstract}

This is an open access article under the CC BY-SA license.

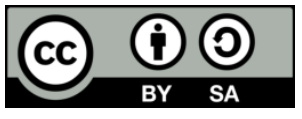

\section{Corresponding Author:}

Abdel-Azim S. Abdel-Salam

Department of Electrical and Computer Engineering,

The Libyan Academy, Tripoli, Libya.

E-mail: abdualadem.saaid@academy.edu.ly

\section{INTRODUCTION}

The motion of a mechanical manipulator described using dynamic equations, these equations are highly non-linear and complex. Therefore, it is very difficult to implement real-time control based on a detailed dynamic model of a robot, the non-linearity of robot manipulator as centrifugal, friction and gravity make a disturbance in this case the conventional control is not effected to control of the robot manipulator. A better solution to the complex control problem might result if human intelligence and judgment replace the design approach of finding an approximation to the true robot system [1].

The fuzzy logic controller can be used to overcome this problem. This is because the performance of fuzzy logic controllers are better than a conventional controller because a fuzzy logic algorithms do not require a detailed mathematical description of the dynamic model to be controlled, and the fuzzy logic controller consider as intelligent controller especially in the non-linear system. Therefore, the implementation of the fuzzy logic controller should be computational less demanding and the position control of a Puma 560 robot manipulator can be achieved by using a fuzzy logic controller. Computed torque control (CTC) is effective with a nonlinear system and uses in robot manipulator for controlling, it is applying feedback linearization to nonlinear system for computes the torque needs for the arm, it works well when all dynamic and physical parameters are known but if the robot has a dynamic parameter variation, then the performance of the controller will not be acceptable [2].

Zadeh in 1965 presented his paper on fuzzy sets and fuzzy logic he was the first one use the theory of the fuzzy set and fuzzy logic [3], and introduce the concept of linguistic variable in 1973, Zadeh showed that fuzzy logic in contrast classical logic may take values between false and true, in classical set theory definition of membership function does not matter, but the number belongs to or does not belong to the set, yes or no and also the zero or one takes on the value, this approach is not appropriate in many life 
applications like the set aged or the set of temperature, but the element in the fuzzy set contains moving values between 0 and 1, meaning that elements of these sets not only represent true or false value but also represent the degree of truth or the degree of falsity of each input [4].

The PUMA 560 robot is an abbreviation for (Programmable Universal Manipulator for Assembly) released in 1978 was the first modern industrial robot and became desperately popular, it is an industrial robot, this robot has 6 degrees of freedom with 6 rotational joints, the Puma 560 was used for research in the 1980s and it was a very common laboratory robot, we used a lot in research because it has been well studied and it is parameters well-known have been described as "white mice" in research robots [5].

\section{THE PUMA 560 ROBOT MODEL}

The specification of the Puma 560 robot manipulator used in this paper is from the paper of Armstrong, Khatib, and Burdick [6]. In this paper, we used the Robotics Toolbox for MATLAB created by Peter Corke in Australia to simulation and implementation of the proposed controllers for control in the position of the Puma 560 robot manipulator [7].

To defined the modified D-H parameters we used this command Puma $560 \mathrm{akb}$ and description creates the robot object used this command P560m, which describes the kinematic and dynamic characteristics of an animation Puma 560 robot manipulators [8]. In this paper, the modified D-H parameters have been used. Craig [9] was the first one used the modified D-H parameters in 1986. The specifications of kinematics taking from the paper of Armstrong, Khatib, and Burdick. In the robotics toolbox, the actuators have been included in the dynamics model of a Puma 560 robot manipulator. Figure 1 shows the Puma 560 robot manipulator according to specification of Armstrong, Khatib and Burdick and used the modified D-H parameter.

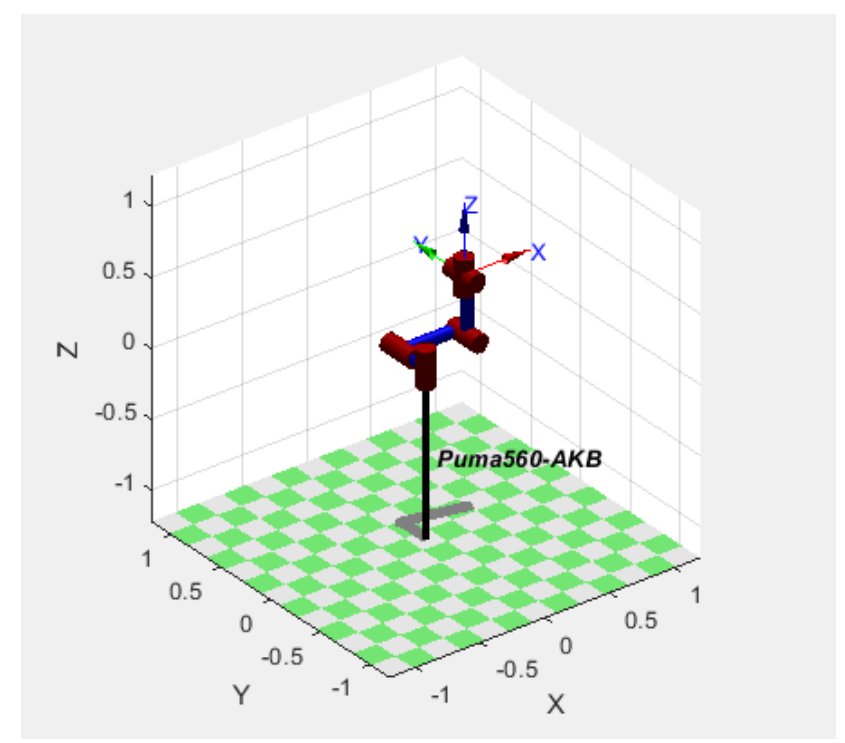

Figure 1. The puma 560-AKB

\section{CONTROLLER DESIGN}

\subsection{PD Computed torque controller}

The motion of the robot describe by (rigid body dynamics):

$$
\tau=M(q) \dot{q}+V(q, \dot{q})+F(q, \dot{q})+G(q)
$$

where;

$M(q) \quad$ Inertia matrix

$V(q, \dot{q}) \quad$ Centrifugal and Coriolis terms

$F(q, \dot{q}) \quad$ Friction term

$G(q) \quad$ Gravity terms

One way to select the control signal $\mathrm{u}(\mathrm{t})$ is as the proportional plus derivative (PD) feedback,

Int J Rob \& Autom, Vol. 9, No. 2, June 2020 : $73-83$ 


$$
u=-k v \dot{e}-k p e
$$

Then the full input for a Puma 560 robot arm (Figure 2) becomes,

$$
\begin{aligned}
& \tau=M(q)(\dot{q} d+k v \dot{e}+k p e)+N(q, \dot{q}) \\
& N(q, \dot{q})=V(q, \dot{q})+F(\dot{q})+G(q)
\end{aligned}
$$

This controller is shown in Figure 3 with $\mathrm{ki}=0$.

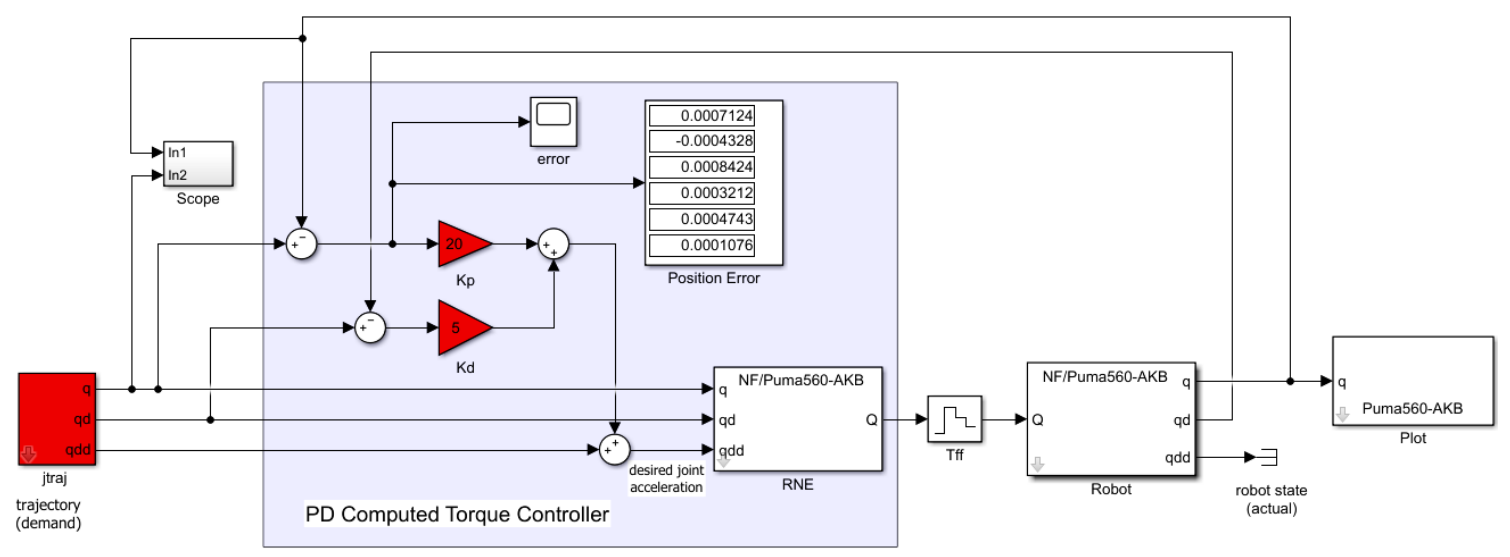

Figure 2. The puma 560 robot Arm with PD-CTC

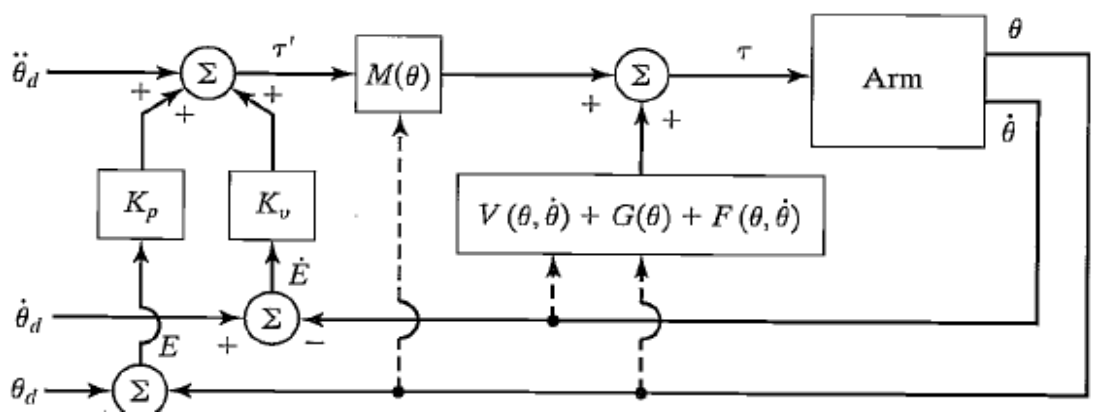

Figure 3. The PD computed torque controller [7]

In this simulation we used some blocks from Peter Corke, Robotics Toolbox, as following:

Jtraj: the purpose of this block is to compute a joint space trajectory between two joint coordinate poses. The command inside the block is:

$$
\text { [q qd qdd]=jtraj(q0,q1,n) }
$$

RNE: the purpose of this block is to compute inverse dynamics by Recursive Newton-Euler method. The command inside the block is:

$$
\text { tau=rne(robot,q,qd,qdd) }
$$

Puma 560-AKB: the purpose of this block is to create a Puma 560 robot. The convention of this robot takes from Armstrong, Khatib, and Burdick. This robot used Craig's modified D-H parameter.

Robot plot: the purpose of this block is graphical robot animation. The command inside the block is:

$$
\text { plot(robot,q) }
$$




\subsection{PID Computed Torque Controller}

We noted PD computed torque control is very effective when all parameters for the arm are known and no disturbance $\tau \mathrm{d}$, from classical control theory if disturbances are constant the PD control gives a nonzero steady-state error, for making the system type (I) we including an integrator in the feedforward loop using PID computed torque controller [10] as in Figure 4. Figure 5 shows The Puma 560 robot arm with PID-CTC.

$$
\begin{aligned}
& \varepsilon^{\cdot}=e \\
& u=-k v \dot{e}-k p e-k i \varepsilon
\end{aligned}
$$

where the arms control becomes as

$$
\tau=M(q)(\dot{q} d+k v \dot{e}+k p e+k i \varepsilon)+N(q, \dot{q})
$$

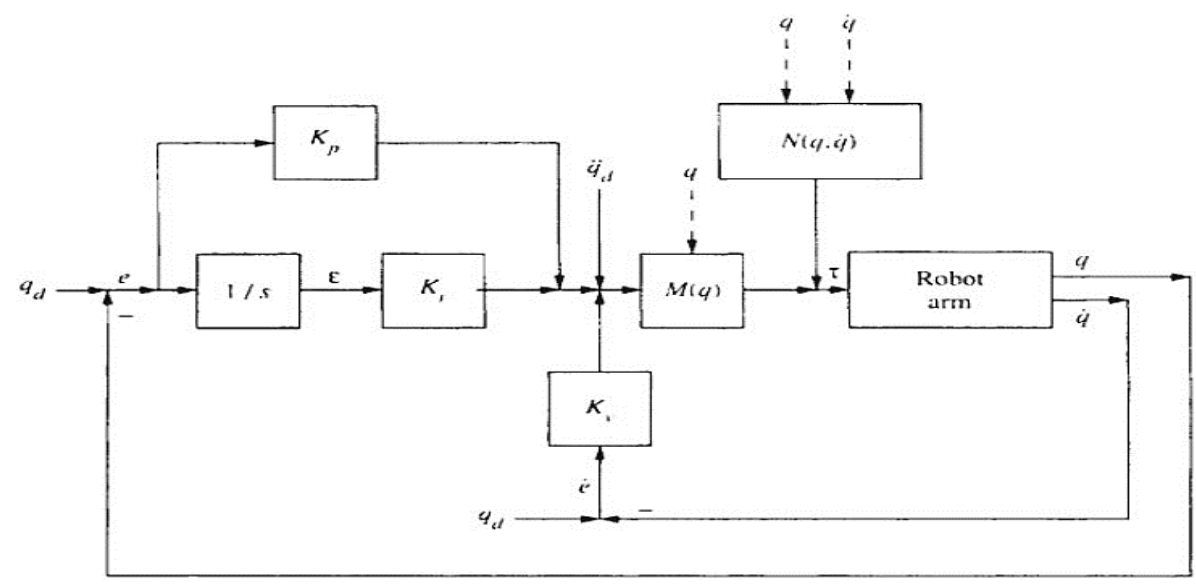

Figure 4. The PID computed torque controller [6]

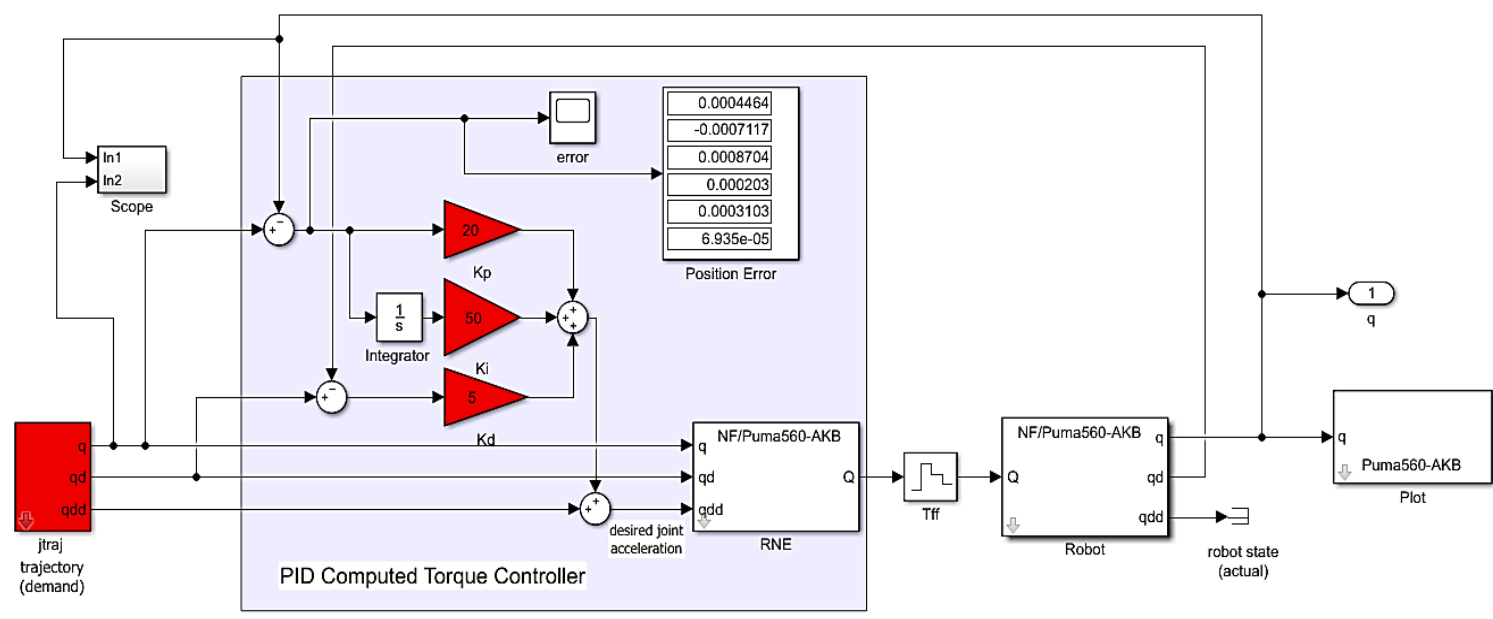

Figure 5. The Puma 560 robot arm with PID-CTC

\subsection{Fuzzy logic controller}

The next steps showing the method to design the fuzzy logic controller.

- Define the input and output to FLC (Figure 6), there are two inputs of FLC, the error $e(t)$ and change of error $\Delta e(t)$ and one output is a control signal $u(t)$ to the plant (Figures 7 and 8).

- Fuzzifying the input and the output variables (Figure 9).

- In the design, we chose 2 input with 7 membership function and 1 output with 7 membership function (Figure 10). 
- In the design, the membership function selected from the negative big $N B$ to the positive big $P B$.

- Chose the inference mechanism rule to find the relation between the input and output, in this paper we used the Mamdani inference mechanism

- Defuzzifying the output variable of the fuzzy mechanism, defuzzification method was used in this paper center of gravity (COG).

NB means Negative Big, NM means Negative Medium, NS means Negative Small, ZE means Zero, PS means Positive Small, PM means Positive Medium and PB means Positive Big. The rule base is in Table 1.

$$
\tau=M(q)(\dot{q} d+\tau f u z z y)+N(q, \dot{q})
$$

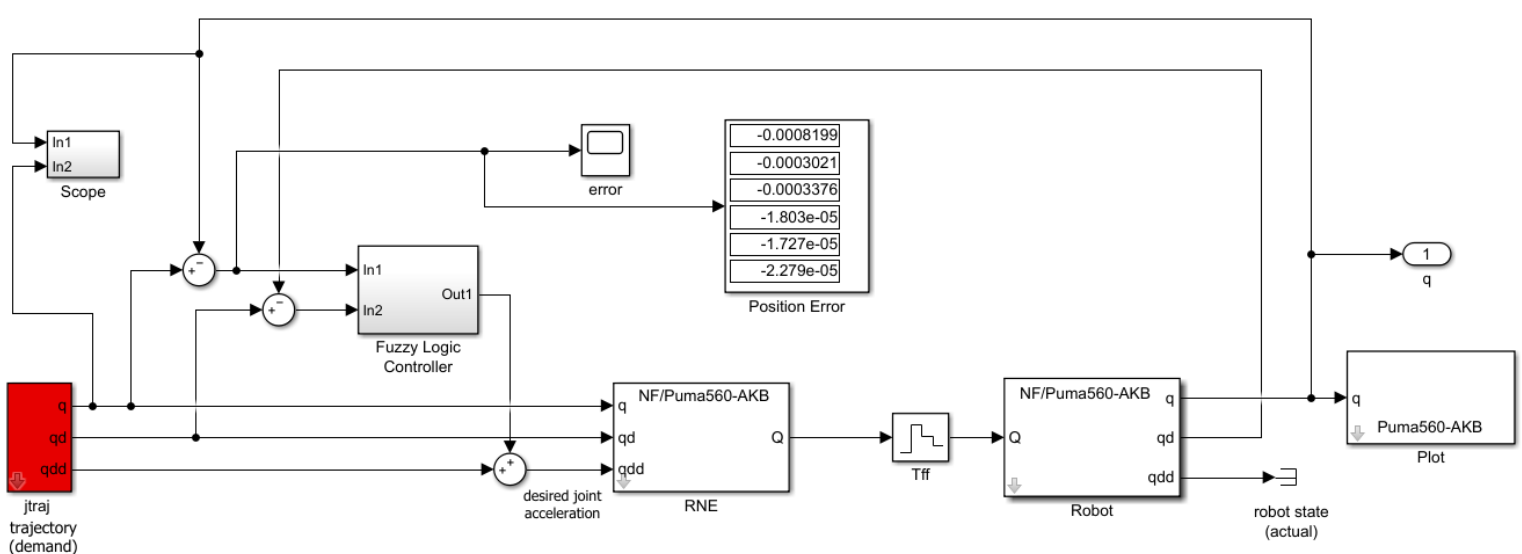

Fuzzy Logic Controller

Figure 6. The puma 560 robot Arm with FLC

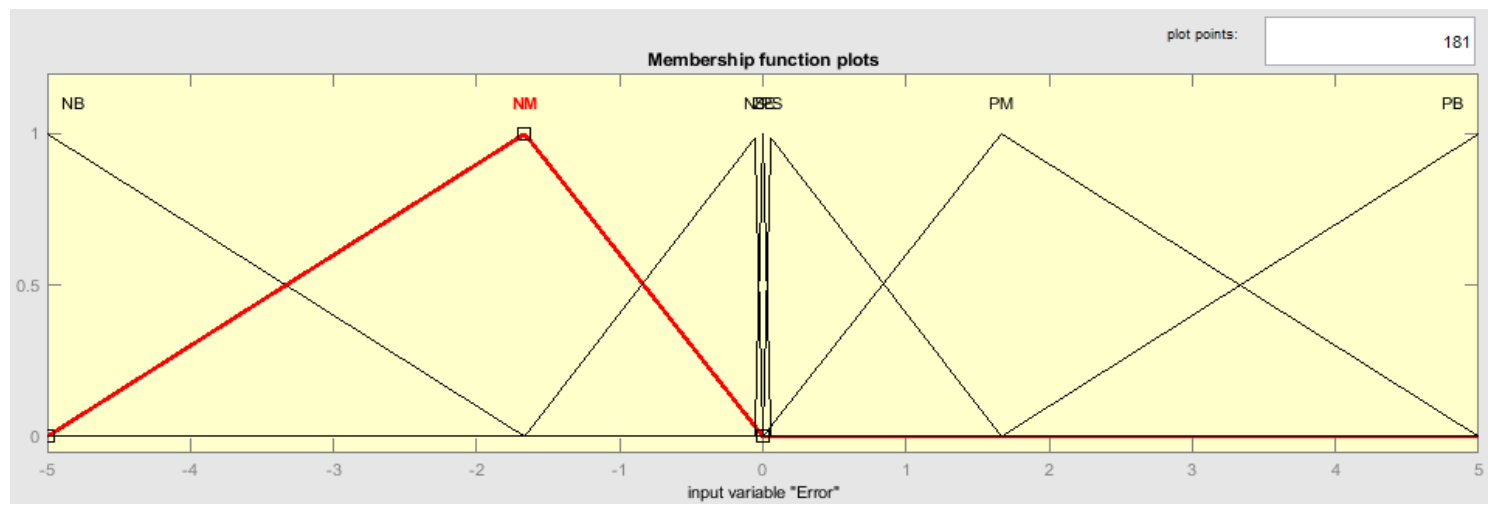

Figure 7. Membership function of $e(t)$

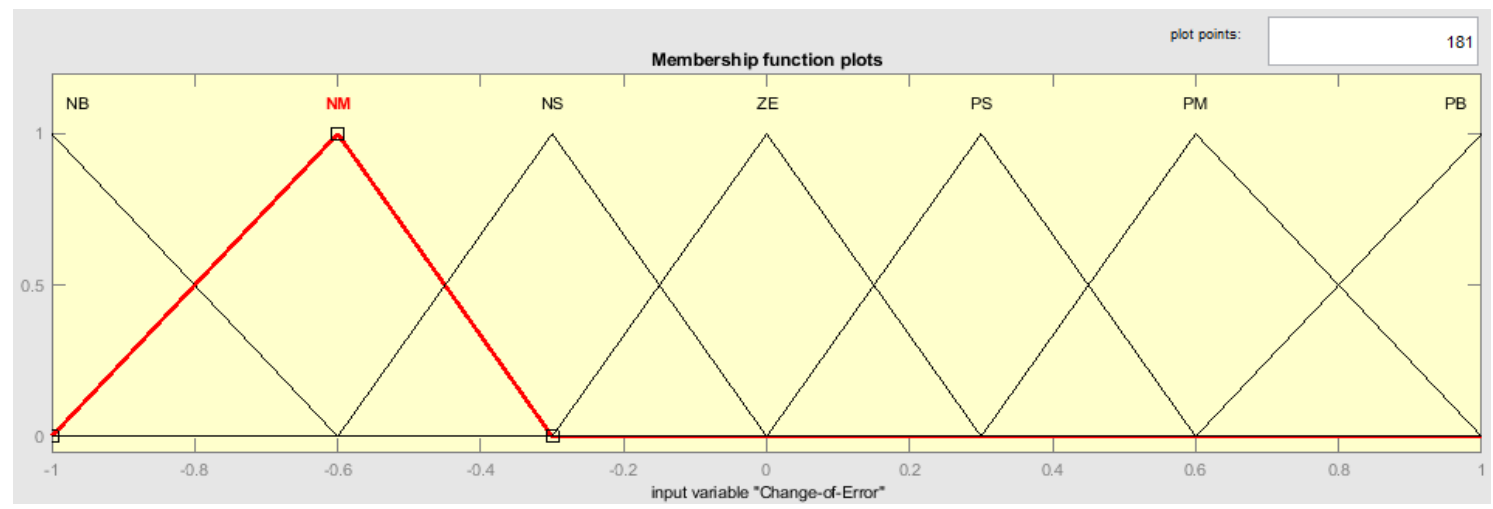

Figure 8. Membership function of $\Delta e(t)$ 


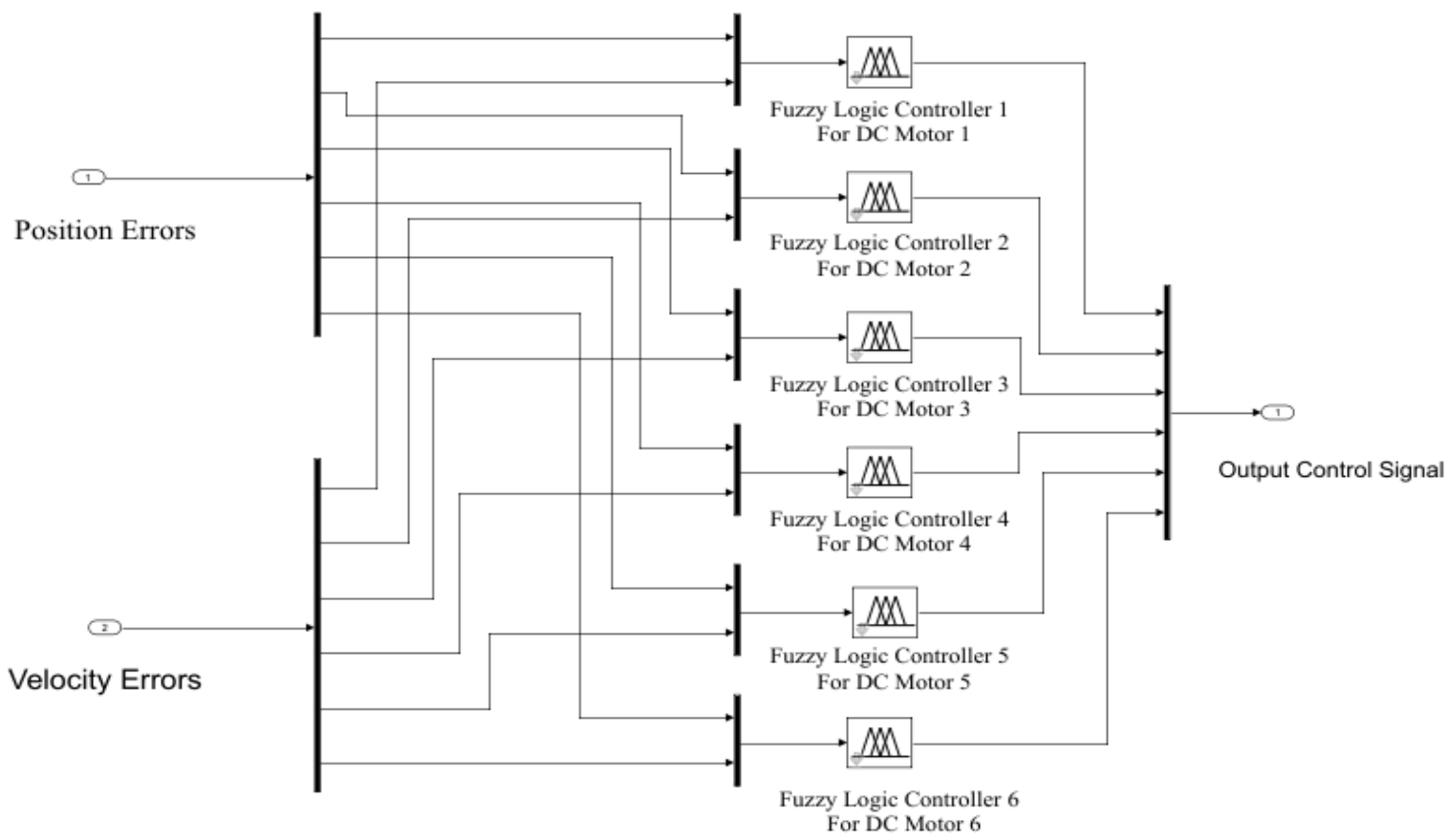

Figure 9. The fuzzy logic controller subsystem

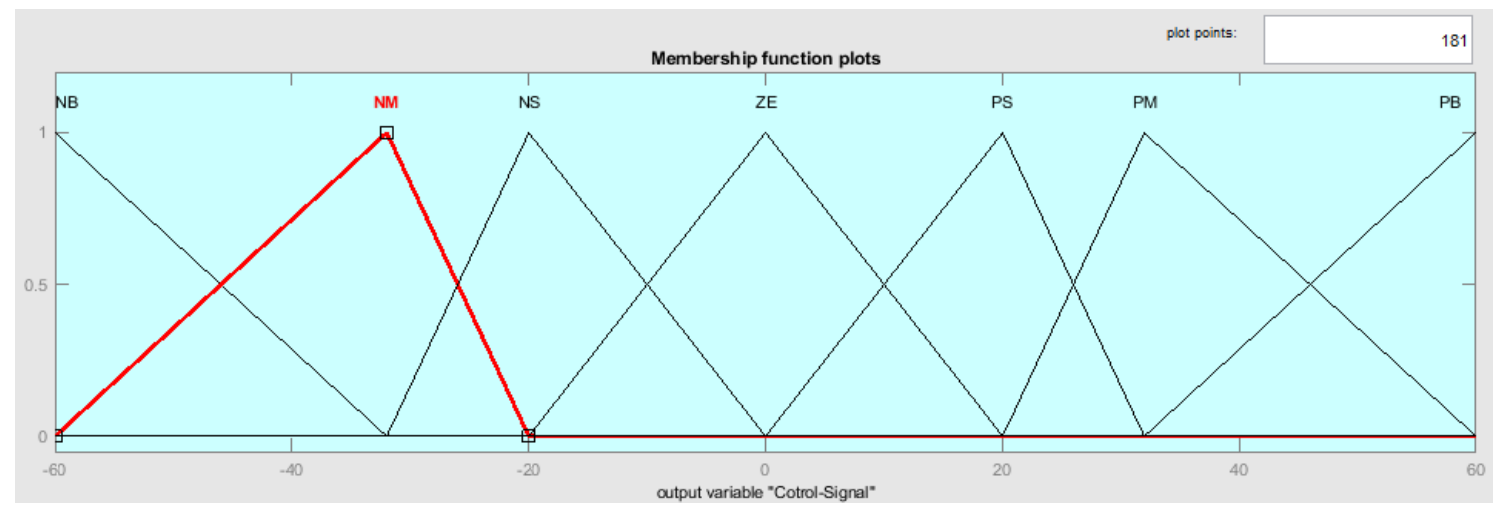

Figure 10. Membership function of output

Table 1. Rule base

\begin{tabular}{ccccccccc}
\hline \multicolumn{1}{c}{ Output } & \multicolumn{7}{c}{ Change of Error } \\
& & NB & NM & NS & ZE & PS & PM & PB \\
\hline \multirow{6}{*}{ Error } & NB & NB & \multicolumn{1}{c}{ NB } & & & NB \\
& NM & NM & NM & & NM & & NM & NM \\
& NS & NS & NS & NS & NS & NS & NS & NS \\
& ZE & NS & NS & NS & ZE & PS & PS & PS \\
& PS & PS & PS & PS & PS & PS & PS & PS \\
& PM & PM & PM & & PM & & & PM \\
& PB & PB & & & PB & & & \\
\hline
\end{tabular}

\section{SIMULATION RESULTS}

To testing the Puma 560 robot manipulator, the joint desired input angles are $\theta$ final $=\left[90^{\circ},-90^{\circ}, 90^{\circ}, 45^{\circ}, 66^{\circ}, 15^{\circ}\right]$ with the initial position of the Puma 560 robot manipulator is the zero position $\theta$ initial $=\left[0^{\circ}, 0^{\circ}, 0^{\circ}, 0^{\circ}, 0^{\circ}, 0^{\circ}\right]$. 


\subsection{Without disturbance}

The performance of PD-CTC, PID-CTC, and FLC without disturbance are good as shown in Figures 11-13, respectively.

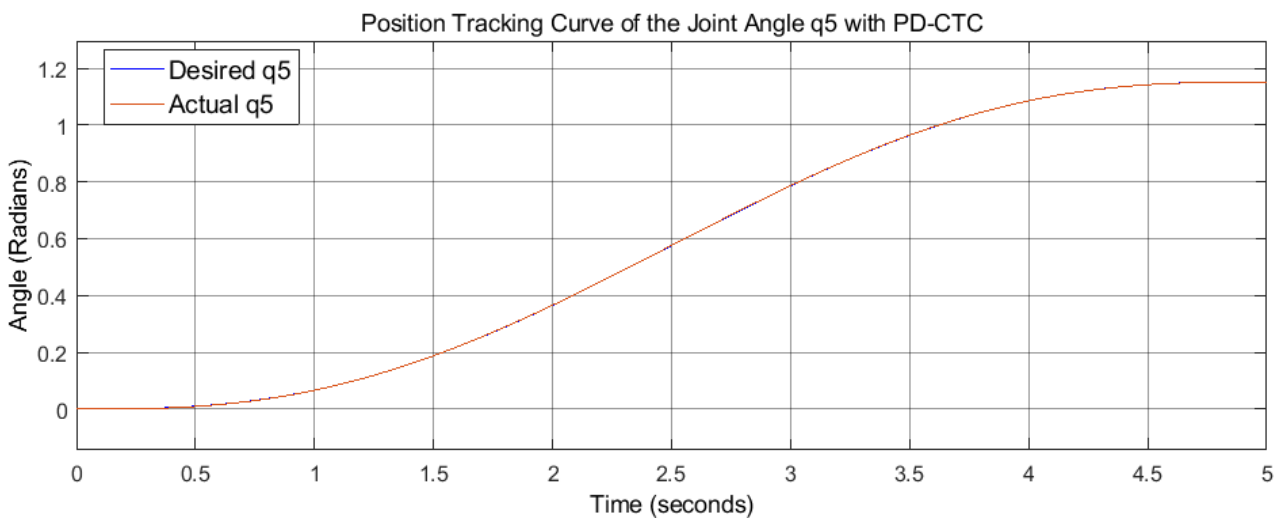

Figure 11. Position tracking curve of joint q5 with PD-CTC

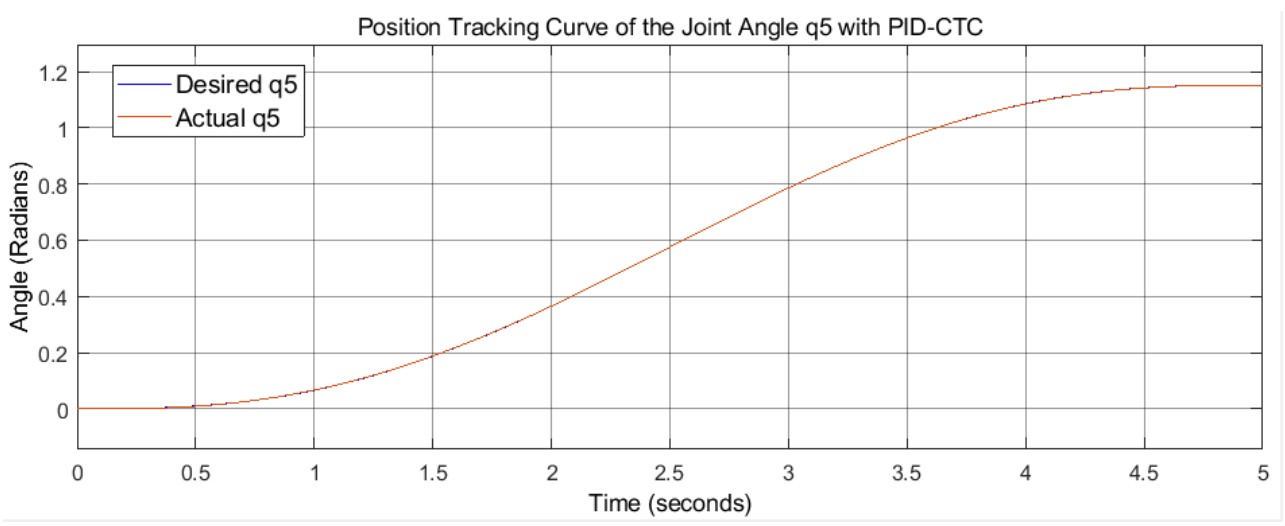

Figure 12. Position tracking curve of joint q5 with PID-CTC

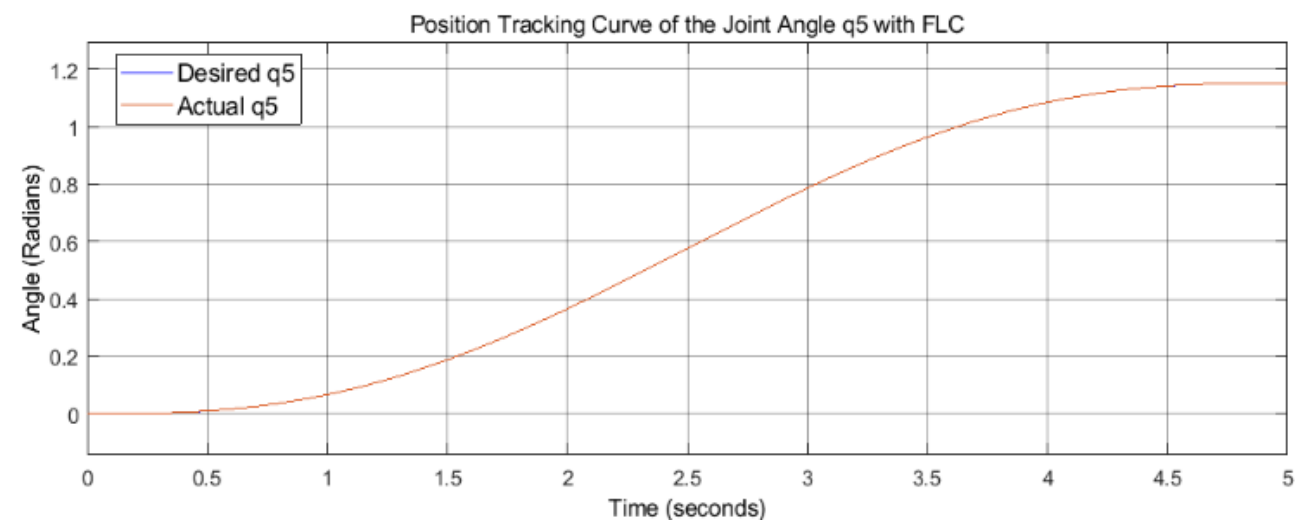

Figure 13. Position tracking curve of joint q5 with FLC

\subsection{With disturbance}

For testing the Puma 560 with disturbance, we chose the maximum torque on each joints for computation the disturbance torque that needs for add to the model, we used the program shows in Figure 14. We take $10 \%$ from the maximum torque to get on the disturbance torque. 


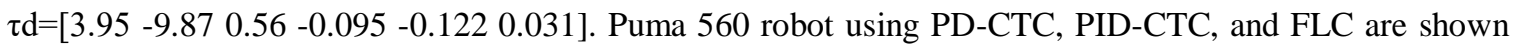
in Figures 15-17 respectively.

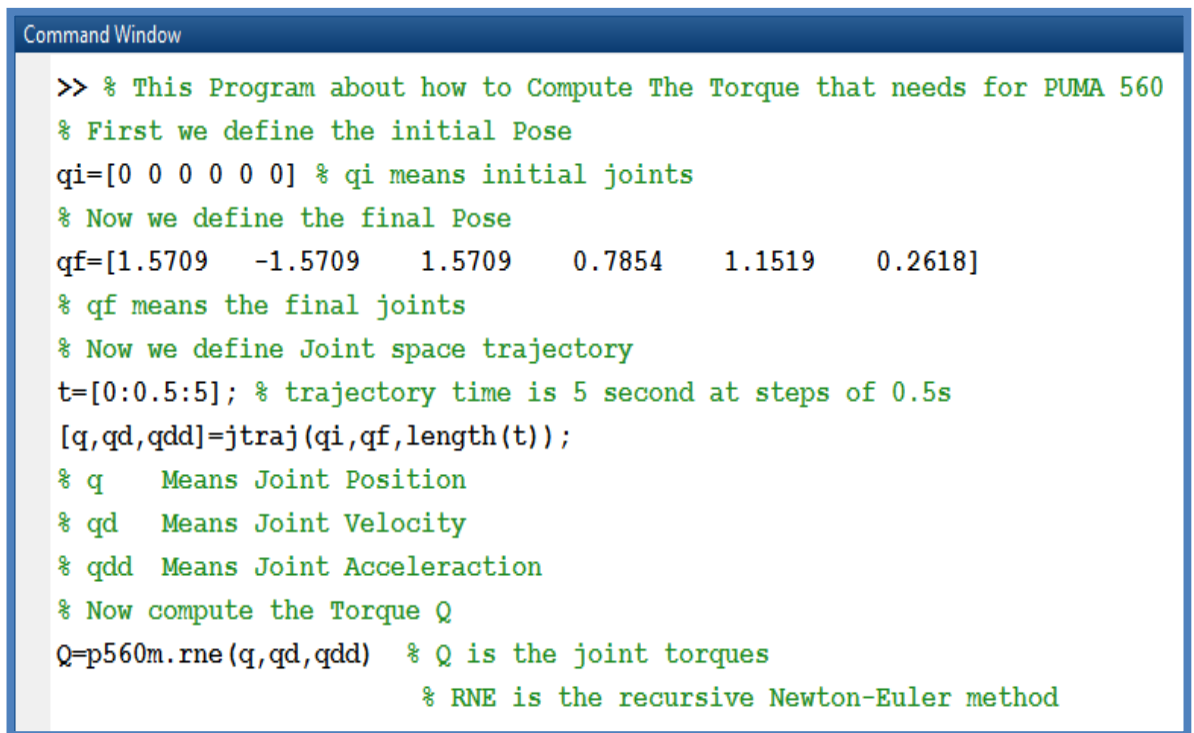

Figure 14. How to compute the torques

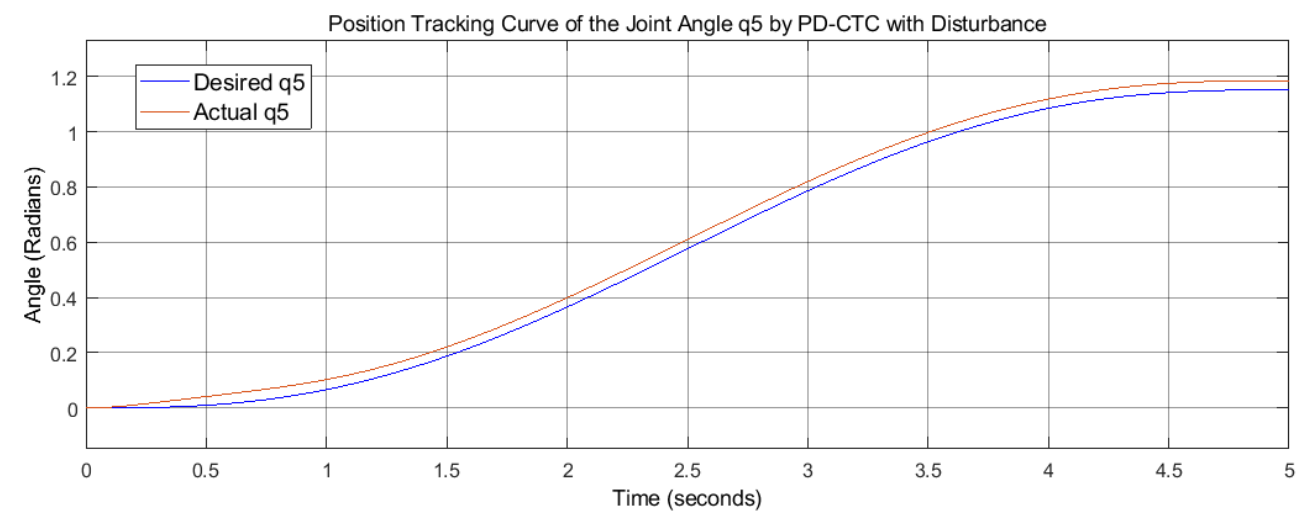

Figure 15. Position tracking curve of joint q5 with PD-CTC

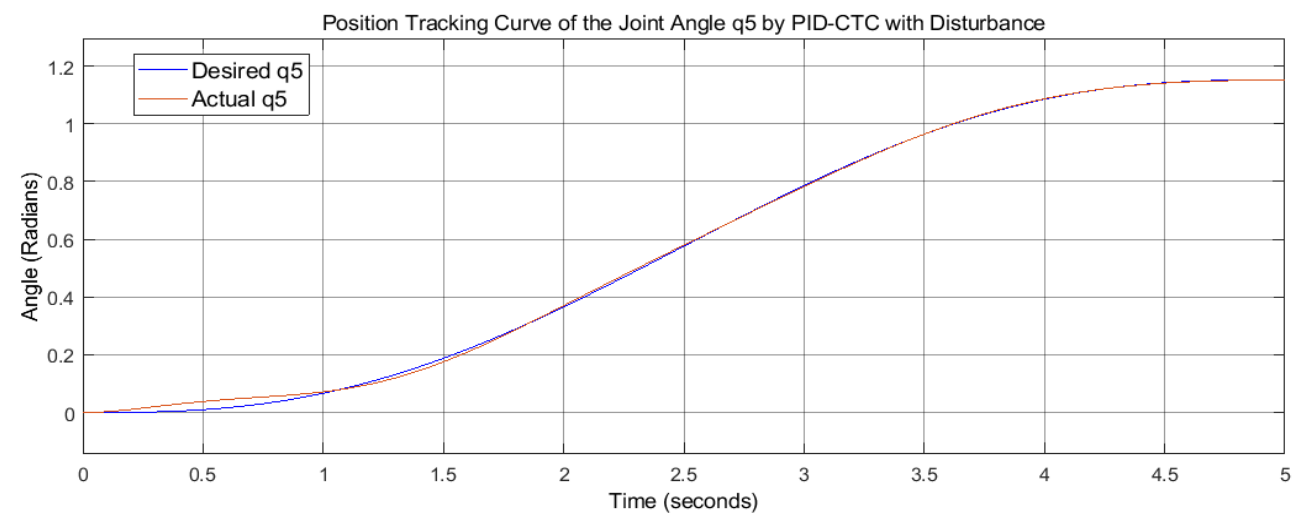

Figure 16. Position tracking curve of joint q5 with PID-CTC 


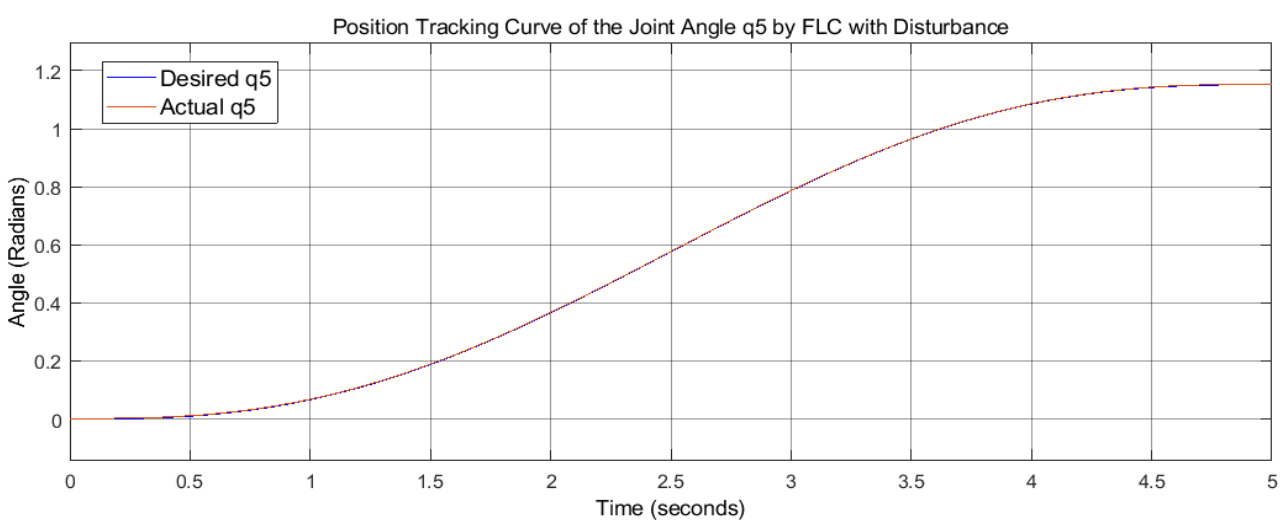

Figure 17. Position tracking curve of joint q5 with FLC

\subsection{With load}

For testing the Puma 560 with load, we added load to the model this load equal $6 \mathrm{~kg}$ using this command

P560m.payload(6, $[0,0,0.1])$

To remove load we used this command

P560m payload (0)

\subsection{The errors position tracking curves}

The positions tracking curve of Puma robot using PD-CTC, PID-CTC, and FLC are shown in Figures 18-20. The Figures 21-23 show the errors signals of the position tracking curves of the controllers PD-CTC, PID-CTC, and FLC with disturbance, respectively.

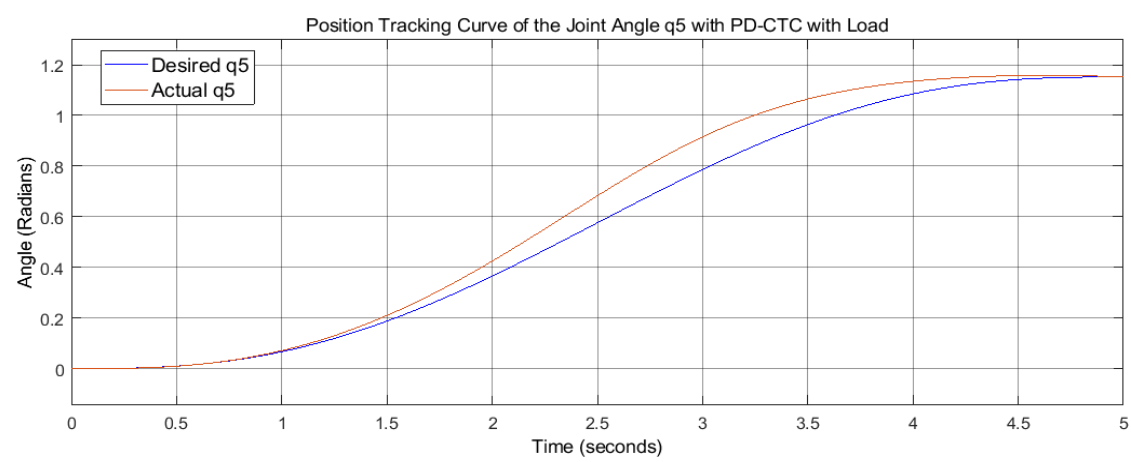

Figure 18. Position tracking curve of joint q5 with PD-CTC

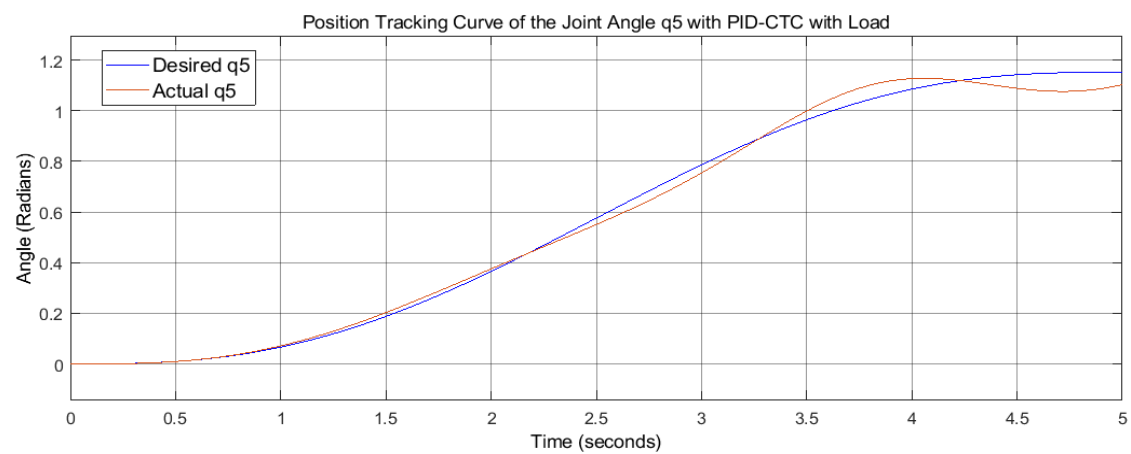

Figure 19. Position tracking curve of joint q5 with PID-CTC 


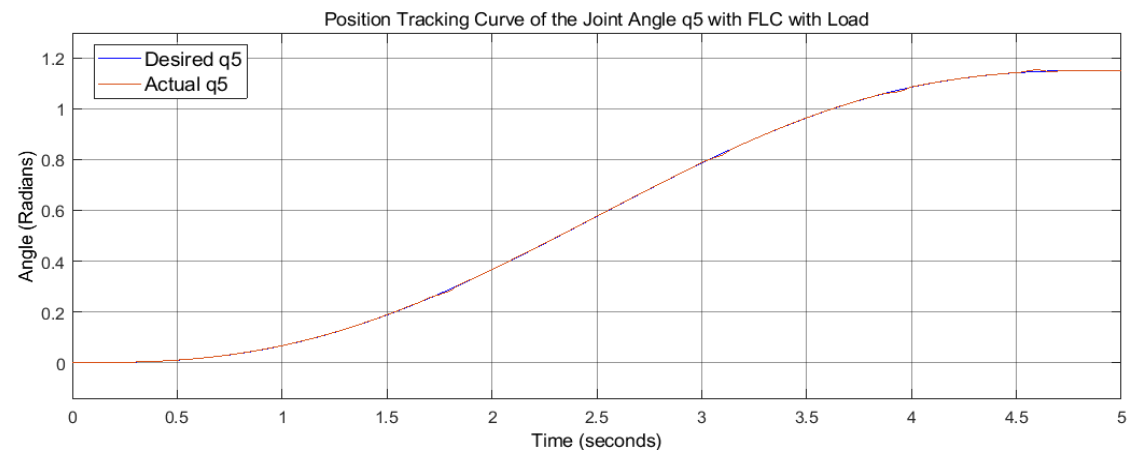

Figure 20. Position tracking curve of joint q5 with FLC

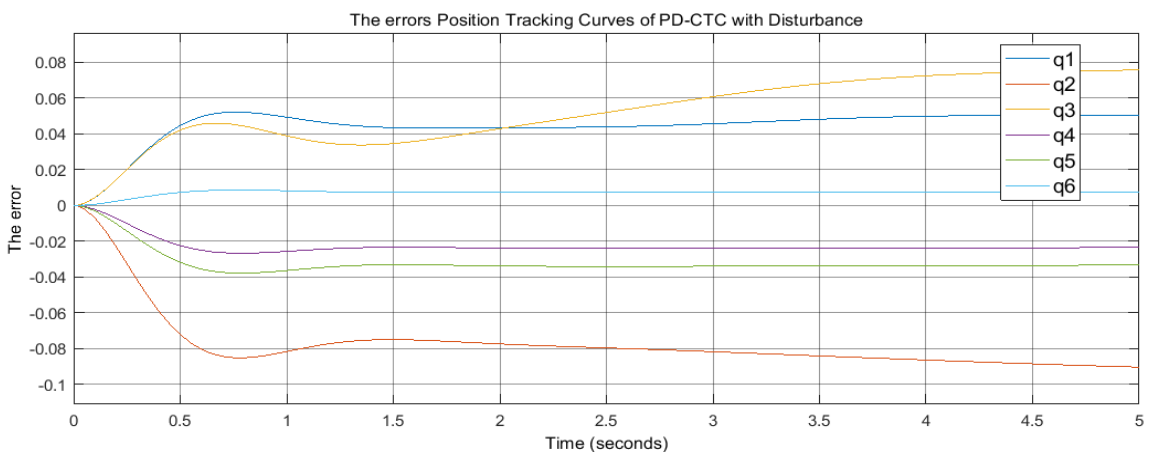

Figure 21. The errors tracking curves of PD-CTC with disturbance

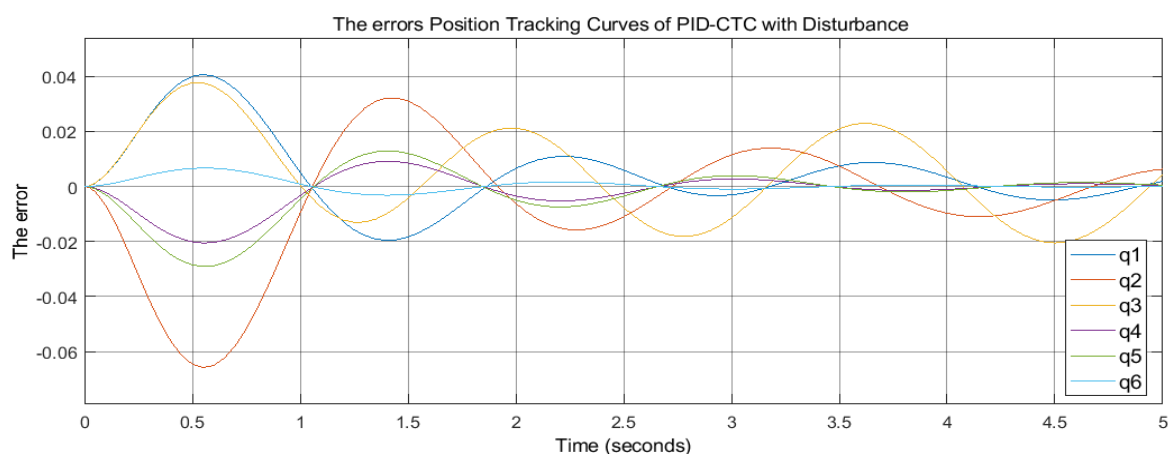

Figure 22. The errors tracking curves of PID-CTC with disturbance

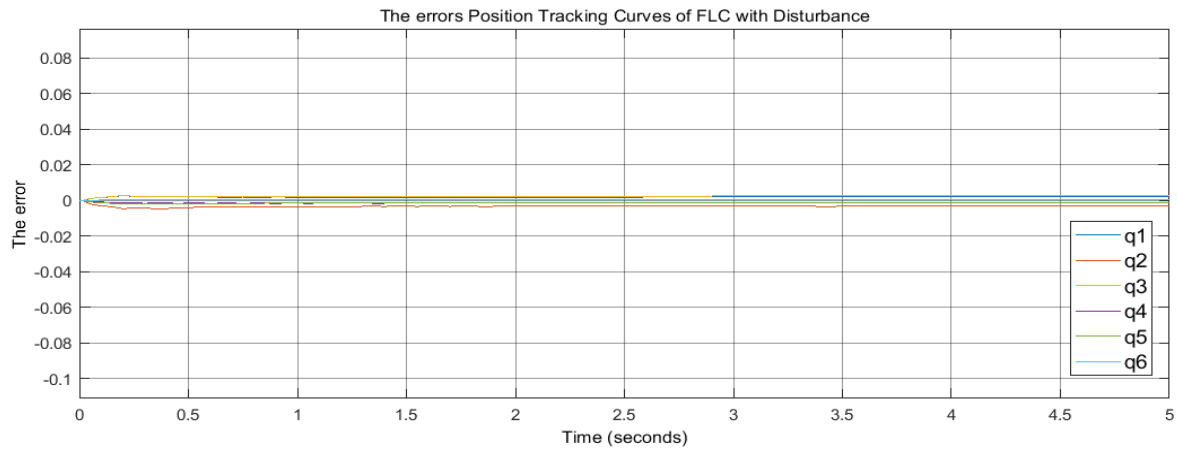

Figure 23. The errors tracking curves of FLC with disturbance 


\section{DISCUSSION}

Note from the simulation that performance of PD-CTC and PID-CTC without disturbance are good, but when add the external disturbance, we note the PD-CTC and PID-CTC are Not good because disturbance torques due to variations in load, coupling and friction that act on the joint. In this case the control problem becomes more difficult and The PD-CTC and PID-CTC are not highly suitable for Nonlinear systems therefore, we used fuzzy logic controller

To overcome on this problem. When we added the disturbance torque the tracking error has increased in the PD-CTC and PID-CTC, but with FLC the tracking performance is good along the path. When we added the load we note that it is affected on the joint 5 and the tracking error has increased in joint 5 in PD-CTC and PID-CTC, but with FLC the joint 5 achieve good tracking performance along the trajectory. From the simulation, we prove that performance of the fuzzy logic controller with disturbance or load is better than another PD-CTC and PID-CTC performance with disturbance or load for controlling in the position of the Puma 560 robot manipulator in terms of position tracking error.

\section{CONCLUSION}

All simulation were presented using MATLAB and Robotics Toolbox (Peter Corke). From the simulation results, we conclude that performance of the fuzzy logic controller in term of position tracking error in case of existence disturbance or load is better than the performance of a computed torque controllers (PD-CTC and PID-CTC).

\section{REFERENCES}

[1] Cubero, S., "Industrial robotics: Theory, modelling and control,” Pro Literatur Verlag, 2006.

[2] Piltan, F., Yarmahmoudi, M. H., Shamsodini, M., Mazlomian, E., and Hosainpour, A., "PUMA-560 robot manipulator position computed torque control methods using Matlab/Simulink and their integration into graduate nonlinear control and Matlab courses," International Journal of Robotics and Automation, vol. 3, no. 3, pp. 167-191, 2012.

[3] Zadeh, L. A., "Fuzzy sets," Information and control, vol. 8, no. 3, pp. 338-353, 1965.

[4] Alassar, A. Z., "Modeling and control of 5 DOF robot arm using supervisory control," in 2010 The 2nd International Conference on Computer and Automation Engineering, vol. 3, pp. 351-355, 2010.

[5] Corke, P., "Robotics, Vision and control: Fundamental algorithms in MATLAB® Second Completely Revised," Springer, vol. 118, 2017.

[6] Armstrong, B., Khatib, O., and Burdick, J., "The explicit dynamic model and inertial parameters of the PUMA 560 arm," Proceedings IEEE International Conference on Robotics and Automation., vol. 3, pp. 510-518, 1986.

[7] Corke, P. I., "A computer tool for simulation and analysis: The robotics toolbox for MATLAB," Proceedings. National Conference Australian Robot Association, pp. 319-330, 1995

[8] Corke, P. I., "A. Robotics toolbox for MATLAB," IEEE Robotics and Automation Magazine, vol. 3, no. 1, pp. 24-32, 2008.

[9] Craig, J. J., "Introduction to robotics: mechanics and control," 3nd Ed., Pearson Prentice Hall, Upper Saddle River, NJ, USA, vol. 3, 2005.

[10] Lewis, F. L., Dawson, D. M., and Abdallah, C. T., "Robot manipulator control: theory and practice," CRC Press, 2003. 